\title{
Wie die Lungenembolie verhindern?
}

\author{
Im letzten Jahr wurde eine neue S2k-Letilinie zur Diagnostik und Thera- \\ pie der Venenthrombose und der Lungenembolie verabschiedet. Das \\ Thema steht im Mittelpunkt der folgenden Gerinnungssprechstunde.
}

Wird eine tiefe Beinvenenthrombose (TVT) diagnostiziert, ist das Gerinnsel ja schon vorhanden. Kann da die Antikoagulation eine Lungenembolie überhaupt noch verhindern, selbst wenn sie sofort eingeleitet wird?

In der Tat kann die Antikoagulation das Risiko, eine Lungenembolie zu entwickeln, nicht auf Null senken. Von dem bereits gebildeten Koagel können sich natürlich Gerinnsel lösen. Wichtig ist aber, durch die sofort eingeleitete Antikoagulation ein Thrombuswachstum zu verhindern, wodurch auch das Lungenembolierisiko drastisch gesenkt wird. Die überwiegende Zahl der Lun-

Tab. 1 Wells-Score zur Ermittlung der klinischen Wahrscheinlichkeit einer Venenthrombose

\begin{tabular}{|c|c|}
\hline Klinische Charakteristika & Score \\
\hline Aktive Tumorerkrankung & 1,0 \\
\hline $\begin{array}{l}\text { Lähmung oder kürzliche } \\
\text { Immobilisation der Beine }\end{array}$ & 1,0 \\
\hline $\begin{array}{l}\text { Bettruhe (> } 3 \text { Tage); große Chirurgie } \\
\text { (<12 Wochen) }\end{array}$ & 1,0 \\
\hline $\begin{array}{l}\text { Schmerz/Verhärtung entlang der } \\
\text { tiefen Venen }\end{array}$ & 1,0 \\
\hline Schwellung ganzes Bein & 1,0 \\
\hline $\begin{array}{l}\text { Unterschenkelschwellung }>3 \mathrm{~cm} \\
\text { gegenüber Gegenseite }\end{array}$ & 1,0 \\
\hline $\begin{array}{l}\text { Eindrückbares Ödem am } \\
\text { symptomatischen Bein }\end{array}$ & 1,0 \\
\hline Kollateralvenen & 1,0 \\
\hline $\begin{array}{l}\text { Frühere, dokumentierte } \\
\text { Venenthrombose }\end{array}$ & 1,0 \\
\hline $\begin{array}{l}\text { Alternative Diagnose mindestens } \\
\text { ebenso wahrscheinlich wie eine } \\
\text { Venenthrombose }\end{array}$ & $-2,0$ \\
\hline \multicolumn{2}{|c|}{$\begin{array}{l}\text { Score <2,0: Wahrscheinlichkeit für TVT nicht hoch; } \\
\text { Score } \geq 2,0: \text { Wahrscheinlichkeit für TVT hoch }\end{array}$} \\
\hline
\end{tabular}

genembolien ereignet sich, bevor die TVT erkannt bzw. behandelt ist.

In Deutschland versterben jährlich ca. 40.000 Menschen an einer Lungenembolie. Die Zahl zeigt, dass bei der Diagnose und Therapie der TVT noch Verbesserungsbedarf besteht.

? Bei der Entstehung der TVT spielen dispositionelle Risikofaktoren eine wichtige Rolle. Wie hoch ist das relative Risiko dieser Faktoren?

Am gefährlichsten ist eine TVT in der Vorgeschichte. Bei solchen Patienten ist das Risiko für ein erneutes Ereignis um das 15-bis 30-Fache erhöht. Durch eine Schwangerschaft wird das Risiko um den Faktor 5-10, bei einem Tumorleiden um den Faktor 7, bei Adipositas um den Faktor 2,5 und bei einer hereditären Trombophilie (APC-Resistenz, Protein C-Mangel etc.) um den Faktor 5 erhöht. \section{3}

Kann mit dem D-Dimer-Test eine TVT immer mit letzter Sicherheit ausgeschlossen werden?

Nein! Ein D-Dimer-Test sollte nur nach vorheriger Einschätzung der klinischen Wahrscheinlichkeit durchgeführt werden. Bei niedriger bzw. mittlerer Wahrscheinlichkeit und normalen D-Dimeren ist keine weitere Thrombosediagnostik nötig, da das Risiko, eine Thrombose zu übersehen, in diesem Fall äußerst gering ist.

Anders ist die Situation bei Patienten mit hoher klinischer Wahrscheinlichkeit. Dazu gehören Patienten mit einem Wells-Score von mindestens 2 (Tab. 1). Bei diesen Patienten ist ein D-DimerTest nicht sinnvoll, es sollte sofort eine weitere Diagnostik mit einer Kompres- sionssonografie erfolgen. Sensitivität und Spezifität dieses Verfahrens liegen bei $>95 \%$.

Muss ein Patient mit TVT Bettruhe einhalten?

Nein! Eine Bettruhe ist in der Regel weder erforderlich noch sinnvoll. Die Therapie kann ambulant erfolgen. Wichtig ist die sofortige Einleitung einer effektiven Antikoagulation. Dabei gibt es heute mehrere Möglichkeiten: Beginn mit einem niedermolekularen Heparin $(\mathrm{NMH})$ oder Fondaparinux und überlappende Einstellung auf einen Vitamin-K-Antagonisten. Als gleichwertige Alternative wird in der neuen S2-Leitlinie ein neues orales Antikoagulans (NOAK) empfohlen. Bei Rivaroxaban und Apixaban kann die Antikoagulation sofort damit begonnen werden. Bei Edoxaban oder Dabigatran sollten die Patienten zunächst über mindestens fünf Tage mit NMH, unfraktionierem Heparin oder Fondaparinux behandelt werden, bevor die Umstellung auf das NOAK erfolgt.

\section{Welchen Stellenwert hat heute noch die Kompression?}

Die Kompressionstherapie ist weiterhin wichtig und sollte deshalb immer durchgeführt werden, da damit das Thrombuswachstum ebenfalls verhindert werden kann. Der besondere Stellenwert des Kompressionsverbandes ergibt sich im Hinblick auf die Verhütung des postthrombotischen Syndroms. Empfehlenswert ist ein Kompressionsstrumpf der Klasse II.

Dr. Peter Stiefelhagen 